Vol. 46 (1992) [179-186]

\title{
A CERTAIN FIXED POINT THEOREM AND ITS APPLICATIONS TO INTEGRAL-FUNCTIONAL EQUATIONS
}

\author{
M. ZIMA
}

In this paper a variant of Banach's contraction principle is established. By using the properties of the spectral radius of a bounded linear operator $A$ defined in a suitable Banach space, we conclude that another operator $\mathcal{A}$ has exactly one fixed point in this space. In the second part of this paper some applications are given.

\section{A CERTAin VARIANT OF Banach'S CONTRACTION PRINCIPLE}

1. BANACH SPACE WITH QUASIMODUlUS. Let $(X,\|\cdot\|, \prec, m)$ denote a Banach space of elements $x \in X$, with a binary relation $\prec$ and a mapping $m: X \rightarrow X$. We shall assume that:

$1^{\circ}$ the relation $\prec$ is transitive,

$2^{\circ} \quad \Theta \prec m(x)$ and $\|m(x)\|=\|x\|$ for all $x \in X$,

$3^{\circ}$ the norm $\|\cdot\|$ is monotonic, that is, if $\Theta \prec x \prec y$ then $\|x\| \leqslant\|y\|$ for all $x, y \in X$.

2. Fixed point theOREM. Now we can formulate the following:

THEOREM 1. In the Banach space considered above, let the operators $\mathcal{A}: X \rightarrow$ $X, A: X \rightarrow X$ be given with the following properties:

$4^{\circ} A$ is a linear bounded operator with the spectral radius $\rho(A)$ less than 1 ,

$5^{\circ} \quad A$ is increasing, that is, if $\theta \prec x \prec y$ then $A x \prec A y$ for all $x, y \in X$,

$6^{\circ} \quad m(\mathcal{A x}-\mathcal{A} y) \prec A m(x-y)$ for all $x, y \in X$.

Then the equation

$$
\mathcal{A} x=x
$$

has a unique solution in the set $X$.

Proof: In the sequel we need the following well known lemma.

Lemma. Lex $X$ be an arbitrary non-empty set and $f: X \rightarrow X$. If there exists $k \in N$ such that $x_{0} \in X$ is the unique solution of the equation $f^{k}(x)=x$, where $f^{k}$

Received 20 August 1991

Copyright Clearance Centre, Inc. Serial-fee code: 0004-9729/92 \$A2.00+0.00. 
denotes $k$ th iteration of the mapping $f$, then $x_{0}$ is the unique solution of the equation $f(x)=x$.

Now, returning to the proof of our theorem, we shall show that there exists a natural number $k$ such that the operator $\mathcal{A}^{k}$ is a strict contraction with respect to the norm $\|\cdot\|$ of the space $(X,\|\cdot\|, \prec, m)$. Indeed, from $5^{\circ}$ and $6^{\circ}$ we get for all $x, y \in X$ :

$$
m\left(\mathcal{A}^{2} x-\mathcal{A}^{2} y\right) \prec A m(\mathcal{A} x-\mathcal{A} y) \prec A^{2} m(x-y)
$$

and generally

$$
m\left(\mathcal{A}^{n} x-\mathcal{A}^{n} y\right) \prec A^{n} m(x-y), \quad x, y \in X, n \in N .
$$

On the other hand, $\rho(A)=\lim _{n \rightarrow \infty} n \sqrt{\left\|A^{n}\right\|}<1$, which means that there exists $k \in N$ such that $\left\|A^{k}\right\|<1$. For this number $k$, in view of (2), we have:

$$
m\left(\mathcal{A}^{k} x-\mathcal{A}^{k} y\right) \prec A^{k} m(x-y)
$$

and consequently, in virtue of $2^{\circ}$ and $3^{\circ}$ :

$$
\begin{aligned}
\left\|m\left(\mathcal{A}^{k} x-\mathcal{A}^{k} y\right)\right\| & =\left\|\mathcal{A}^{k} x-\mathcal{A}^{k} y\right\| \leqslant\left\|A^{k} m(x-y)\right\| \\
& \leqslant\left\|A^{k}\right\|\|m(x-y)\|=\left\|A^{k}\right\|\|x-y\| .
\end{aligned}
$$

Finally we get

$$
\left\|\mathcal{A}^{k} x-\mathcal{A}^{k} y\right\| \leqslant\left\|A^{k}\right\|\|x-y\| \quad \text { for each } \quad x, y \in X .
$$

Since $\left\|A^{k}\right\|<1$, the operator $\mathcal{A}^{k}$ is a strict contraction in the space $(X,\|\cdot\|, \prec, m)$ and, by Banach's contraction principle, the equation

$$
\mathcal{A}^{k} x=x
$$

has a unique solution in the set $X$. In virtue of the lemma the equation $\mathcal{A} x=x$ has a unique solution in the set $X$ too. This completes the proof of Theorem 1.

\section{SOME APplications of Theorem 1}

1. The integral-functional equation. Let $X$ denote a set of real continuous functions on the interval $[0, T]$. We shall define a relation $\prec$ as follows: $x \prec y$ if and only if $x(t) \leqslant y(t)$ for each $t \in[0, T]$. Moreover, let $m(x)=|x|$, that is, $(m(x))(t)=$ $|x(t)|$ for $t \in[0, T]$ and $\|x\|=\max _{[0, T]}|x(t)|$.

It is easy to see that the conditions $1^{\circ}-3^{\circ}$ are satisfied in this case. 
We shall consider the following operator:

$$
(\mathcal{A} x)(t)=f(t)+\int_{0}^{t} F(s, x(h(s))) d s, \quad t \in[0, T]
$$

assuming that:

$7^{\circ} \quad F:[0, T] \times R \rightarrow R$ is continuous and satisfies the inequality

$$
|F(t, x)-F(t, y)| \leqslant L(t)|x-y|,
$$

where the function $L$ is positive and continuous on $[0, T]$;

$8^{\circ} f:[0, T] \rightarrow R$ and $h:[0, T] \rightarrow[0, T]$ are continuous too.

The condition $4^{\circ}$ of Theorem 1 becomes

$$
\begin{gathered}
\left|\int_{0}^{t} F(s, x(h(s))) d s-\int_{0}^{t} F(s, y(h(s))) d s\right| \\
\leqslant \int_{0}^{t} L(s)|x(h(s))-y(h(s))| d s
\end{gathered}
$$

and, in virtue of $7^{\circ}$, is satisfied.

The operator $A$ assumes the form:

$$
(A x)(t)=\int_{0}^{t} L(s) x(h(s)) d s, \quad t \in[0, T] .
$$

Now we can formulate the following:

Theorem 2. If the conditions $7^{\circ}-8^{\circ}$ are satisfied and the spectral radius of operator (8) is less than 1 , then the equation

$$
x(t)=f(t)+\int_{0}^{t} F(s, x(h(s))) d s, \quad t \in[0, T]
$$

has exactly one solution in the set of continuous functions on $[0, T]$.

2. Calculation of the spectral radius of operator (8). Let $A^{n}$ denote the $n$th iteration of operator (8). Then we get the following formula:

$$
\left(A^{n} x\right)(t)=\int_{0}^{t} L(s)\left(A^{n-1} x\right)(h(s)) d s, \quad t \in[0, T], \quad n=1,2, \ldots
$$

Let $K$ denote a cone of continuous non-negative functions on $[0, T]$ (see for example $[3,4])$. Since int $K \neq \emptyset$ and $K$ is normal, we can calculate the spectral radius of operator (8) by the formula [4]:

$$
\rho(A)=\lim _{n \rightarrow \infty}\left(\left\|A^{n} x_{0}\right\|\right)^{1 / n},
$$


where $x_{0}$ is a suitably chosen element of $K$. In our case we may take $x_{0}(t) \equiv 1$ on $[0, T]$. Hence

$$
\left\|A^{n} x_{0}\right\|=\int_{0}^{T} L(s)\left(A^{n-1} x_{0}\right)(h(s)) d s=\left(A^{n} x_{0}\right)(T)
$$

If, for every $n \in N,\left\|A^{n} x_{0}\right\|>0$ and there exists

$$
\lim _{n \rightarrow \infty} \frac{\left\|A^{n+1} x_{0}\right\|}{\left\|A^{n} x_{0}\right\|}
$$

then

$$
\rho(A)=\lim _{n \rightarrow \infty} \sqrt[n]{\left\|A^{n} x_{0}\right\|}=\lim _{n \rightarrow \infty} \frac{\left\|A^{n+1} x_{0}\right\|}{\left\|A^{n} x_{0}\right\|} .
$$

In view of (11) and (12) we get:

$$
\rho(A)=\lim _{n \rightarrow \infty} \frac{\left(A^{n+1} x_{0}\right)(T)}{\left(A^{n} x_{0}\right)(T)}
$$

Particularly, if $L(t)=L t^{\alpha}, h(t)=t^{\beta}, L>0, \alpha>0, \beta>0, T=1$, then

$$
(A x)(t)=L \int_{0}^{t} s^{\alpha} x\left(s^{\beta}\right) d s, \quad t \in[0,1]
$$

so

$$
\begin{aligned}
\left(A x_{0}\right)(t) & =L \int_{0}^{t} s^{\alpha} d s=\frac{L t^{\alpha+1}}{\alpha+1} \\
\left(A^{2} x_{0}\right)(t) & =\frac{L^{2} t^{\beta(\alpha+1)+\alpha+1}}{(\alpha+1)[\beta(\alpha+1)+\alpha+1]}
\end{aligned}
$$

and generally

$$
\begin{gathered}
\left(A^{n} x_{0}\right)(t)=\frac{L^{n} t^{a_{n}}}{a_{1} a_{2} \ldots a_{n}}, \text { where } a_{1}=\alpha+1, a_{k}=\beta a_{k-1}+a_{1} \\
k=2,3, \ldots
\end{gathered}
$$

Hence

and

$$
\begin{gathered}
\left(A^{n} x_{0}\right)(T)=\left(A^{n} x_{0}\right)(1)=\frac{L^{n}}{a_{1} a_{2} \ldots a_{n}} \\
\frac{\left(A^{n+1} x_{0}\right)(1)}{\left(A^{n} x_{0}\right)(1)}=\frac{L}{a_{n+1}}=\frac{L}{(\alpha+1)\left(1+\beta+\ldots+\beta^{n}\right)}
\end{gathered}
$$


so in the case $L(t)=L t^{\alpha}, h(t)=t^{\beta}$, the spectral radius $\rho(A)$ of operator (8) has the value

$$
\rho(A)=\frac{L}{\alpha+1} \cdot \frac{1}{\sum_{n=0}^{\infty} \beta^{n}} .
$$

If $\beta \geqslant 1$, then $\sum_{n=0}^{\infty} \beta^{n}$ is divergent and $\rho(A)=0$. It corresponds to the situation when the function $h(t)$ does not advance the variable $t$ on the interval $[0, T]$. If $0<\beta<1$ then

$$
\rho(A)=\frac{L(1-\beta)}{\alpha+1}
$$

REMARK. In the paper [7] for $\alpha=0, \beta=1 / 2$ we obtained the following result:

If $L<4 / e$ then the operator

$$
(A x)(t)=\int_{0}^{t} L x(\sqrt{s}) d s, \quad t \in[0,1]
$$

is a strict contraction in the suitable space. It follows from (13) that if only $L<2$ then the equation

$$
x(t)=f(t)+\int_{0}^{t} L x(\sqrt{s}) d s, \quad t \in[0,1]
$$

has exactly one solution in the space of continuous functions on $[0,1]$.

3. The initial value problem of neutral type. Now we shall consider the following initial value problem:

$$
\begin{aligned}
& x^{\prime}(t)=f\left(t, x\left(h_{1}(t)\right), x\left(h_{2}(t)\right), \ldots, x\left(h_{r}(t)\right), x^{\prime}(t)\right), t \in[0, T] \\
& x(0)=0 .
\end{aligned}
$$

Suppose that:

$9^{\circ} f:[0, T] \times R^{r+1} \rightarrow R$ is continuous and satisfies the Lipschitz condition: for all $\left(t, x_{1}, \ldots, x_{r+1}\right),\left(t, y_{1}, \ldots, y_{r+1}\right) \in[0, T] \times R^{r+1}$ $\left|f\left(t, x_{1}, \ldots, x_{r+1}\right)-f\left(t, y_{1}, \ldots, y_{r+1}\right)\right| \leqslant \sum_{i=1}^{r+1} L_{i}(t)\left|x_{i}-y_{i}\right|$, where the functions $L_{i}, i=1,2, \ldots, r+1$, are continuous and positive on the interval $[0, T]$;

$10^{\circ} h_{i}:[0, T] \rightarrow[0, T]$ are continuous, $i=1,2, \ldots, r$. 
THEOREM 3. Let the conditions $9^{\circ}-10^{\circ}$ be satisfied. If $h_{i}(t) \leqslant t$ for $t \in[0, T]$, $i=1,2, \ldots, r$ and $\max _{[0, T]}\left\{L_{r+1}(t)\right\}<1$ then the problem (14) has exactly one solution in the set of continuous functions on $[0, T]$.

Proof: Let us notice that the problem (13) is equivalent to the functional-integral equation

$$
z(t)=f\left(t, \int_{0}^{h_{1}(t)} z(s) d s, \ldots, \int_{0}^{h_{r}(t)} z(s) d s, z(t)\right), \quad t \in[0, T]
$$

where $z(t)=x^{\prime}(t)$ on $[0, T]$.

To prove our theorem it is sufficient to show that the operator

$$
(\mathcal{A} z)(t)=f\left(t, \int_{0}^{h_{1}(t)} z(s) d s, \ldots, \int_{0}^{h_{r}(t)} z(s) d s, z(t)\right), t \in[0, T]
$$

has a unique fixed point in the set of continuous functions.

From $9^{\circ}-10^{\circ}$ it follows that

$$
\begin{aligned}
|(\mathcal{A} z)(t)-(\mathcal{A} w)(t)|= & \mid f\left(t, \int_{0}^{h_{1}(t)} z(s) d s, \ldots, \int_{0}^{h_{r}(t)} z(s) d s, z(t)\right) \\
& -f\left(t, \int_{0}^{h_{1}(t)} w(s) d s, \ldots, \int_{0}^{h_{r}(t)} w(s) d s, w(t)\right) \mid \\
\leqslant & \sum_{i=1}^{r} L_{i}(t) \int_{0}^{h_{i}(t)}|z(s)-w(s)| d s+L_{r+1}(t)|z(t)-w(t)| \\
\leqslant & L \sum_{i=1}^{r} \int_{0}^{h_{i}(t)}|z(s)-w(s)| d s+L_{r+1}(t)|z(t)-w(t)|,
\end{aligned}
$$

where $L=\max _{1 \leqslant i \leqslant r}\left(\max _{[0, T]}\left\{L_{i}(t)\right\}\right)$.

Let $L_{r+1}=\max _{[0, T]}\left\{L_{r+1}(t)\right\}$. Since $h_{i}(t) \leqslant t$ on $[0, T], i=1,2, \ldots, r$ we obtain:

$$
|(\mathcal{A} z)(t)-(\mathcal{A} w)(t)| \leqslant L r \int_{0}^{t}|z(s)-w(s)| d s+L_{r+1}|z(t)-w(t)| .
$$

Let us denote

$$
\left(A_{1} u\right)(t)=L r \int_{0}^{t} u(s) d s, \quad\left(A_{2} u\right)(t)=L_{r+1} u(t) .
$$

It is easy to see that

$$
A:=A_{1}+A_{2}
$$


is a linear bounded and increasing operator.

We can write (16) as

$$
|(\mathcal{A} z)(t)-(\mathcal{A} w)(t)| \leqslant(A|z-w|)(t) .
$$

It means that the assumption $6^{\circ}$ of Theorem 1 is satisfied. Since the operator $A_{1}$ is of Volterra type, $\rho\left(A_{1}\right)=0$. It is easy to check by elementary calculation that $\rho\left(A_{2}\right)=$ $L_{r+1}$. Moreover the operators $A_{1}, A_{2}$ are commutative, that is, $A_{1} A_{2}=A_{2} A_{1}$. Thus, by XI.21 of [5]:

$$
\rho\left(A_{1}+A_{2}\right) \leqslant \rho\left(A_{1}\right)+\rho\left(A_{2}\right) .
$$

From (17) and (18) we have:

$$
\rho(A) \leqslant L_{r+1} .
$$

Since $L_{r+1}<1$, by virtue of Theorem 1 , the operator (15) has exactly one fixed point in the set of continuous functions on $[0, T]$. This completes the proof of Theorem 3.

REMARK. The problem (14) is similar to the initial value problems considered in the papers $[1,2,6]$. The direct application of the uniqueness condition from [6] to the problem (14) leads us to the assumption

$$
\frac{\sum_{i=1}^{r} L_{i}}{l}+L_{r+1}<1,
$$

where $L_{i}=\max _{[0, T]}\left\{L_{i}(t)\right\}, i=1,2, \ldots, r$, and $l$ is a positive number.

Thanks to the applied method, the uniqueness condition in our paper is clearly better than (19). It is noteworthy that the uniqueness of solution of (14) does not depend on the coefficients $L_{i}(t), i=1,2, \ldots, r$, but on $L_{r+1}(t)$ only.

\section{REFERENCES}

[1] J. Banaś, 'Applications of measures of noncompactness to various problems', Folia Scientiarum Universitatis Technicae Resoviensis 34 (1987).

[2] S. Czerwik, 'Existence, uniqueness and continuous dependence for the parameter of solutions of a system of differential equations with deviating argument', Ann. Polon. Math. 34 (1977), 269-275.

[3] K. Deimling, Nonlinear functional analysis (Springer-Verlag, Berlin, Heidelberg, New York, Tokyo, 1985).

[4] M.A. Krasnoselski et al, Näherungsverfahren zur Lösung von Operatorgleichungen (Akademie-Verlag, Berlin, 1973). 
[5] F. Riesz and B. Sz.-Nagy, Leçons d'analyse fonctionelle, (in Russian) (Mir Moscow, 1970).

[6] P. Ch. Tsamatos, 'Existence and uniqueness of solutions of neutral type differential equations', Fasc. Math. 14 (1985), 63-72.

[7] M. Zima, 'On the existence and uniqueness of solution of certain initial value problem', Folia Scientiarum Universitatis Technicae Resoviensis 48 (1988), 113-118.

\author{
Department of Mathematics \\ Pedagogical University of Rzeszów \\ Rzeszów \\ Poland
}

